\title{
Factors influencing negative surgical outcomes in adult pectus excavatum patients undergoing Nuss procedure
}

\author{
Zhen Gao ${ }^{1,2 \#}$, Shujie Huang ${ }^{1,3 \#}$, Yong Tang $^{1}$, Sichao Wang ${ }^{1}$, Weitao Zhuang ${ }^{1,3}$, Yu Ding ${ }^{1,2}$, Hansheng Wu ${ }^{1}$, \\ Jiming Tang ${ }^{1}$, Dongkun Zhang ${ }^{1}$, Haiyu Zhou ${ }^{1}$, Xiaosong Ben ${ }^{1}$, Liang Xie ${ }^{1}$, Gang Chen ${ }^{1}$, Guibin Qiao ${ }^{1}$ \\ ${ }^{1}$ Department of Thoracic Surgery, Guangdong Provincial People's Hospital, Guangdong Academy of Medical Sciences, Guangzhou, China; ${ }^{2}$ The \\ Second School of Clinical Medicine, Southern Medical University, Guangzhou, China; ${ }^{3}$ Shantou University Medical College, Shantou, China \\ Contributions: (I) Conception and design: Z Gao, S Huang, Y Tang, G Qiao; (II) Administrative support: G Qiao, G Chen; (III) Provision of study \\ materials or patients: Z Gao, S Huang; (IV) Collection and assembly of data: Z Gao, S Huang; (V) Data analysis and interpretation: All authors; (VI) \\ Manuscript writing: All authors; (VII) Final approval of manuscript: All authors. \\ \#These authors contributed equally to this work. \\ Correspondence to: Guibin Qiao, MD, PhD. Department of Thoracic Surgery, Guangdong Provincial People's Hospital, Guangdong Academy of \\ Medical Sciences, Zhongshan Second Road, Guangzhou 510080, China. Email: guibinqiao@126.com.
}

Background: This study aimed to examine the factors associated with the negative outcomes of Nuss procedure in adult pectus excavatum (PE) patients.

Methods: Forty-seven adult PE patients were enrolled in this study. Mimics 21.0 software (Materialise) was used to reconstruct the preoperative and postoperative three-dimensional (3D) thoracic model. The preoperative and postoperative pulmonary volumes and function parameters were compared. The diaphragm positions were localized, and the anteroposterior diameter (APD) of the thoracic cavity was calculated using neoteric methods. Binary logistic regression was used to reveal the association between clinical factors and altered pulmonary parameters.

Results: Postoperative lung volumes in adult $\mathrm{PE}$ patients decreased significantly $(\mathrm{P}<0.001)$. The mean preoperative lung volume was $4,592.82 \pm 946.54 \mathrm{~cm}^{3}$, which reduced to $3,976.26 \pm 867.35 \mathrm{~cm}^{3}$ postoperatively. The rate of postoperative lung volume reduction was approximately $12.1 \%$. Physiologically, forced expiratory volume in 1 second (FEV1), forced vital capacity (FVC), and peak expiratory flow (PEF) significantly decreased after Nuss procedure, and a near 10\% reduction in FVC was observed. Diaphragm elevation was positively associated with decrease in lung volumes [odds ratio $(\mathrm{OR})=40.51 ; \mathrm{P}=0.011 ; 95 \%$ confidence interval (CI), 2.37-692.59]. The presence of reduced thoracic APDs was significantly associated with negative pulmonary function results ( $\mathrm{OR}=1.21$; $\mathrm{P}=0.008$; $95 \% \mathrm{CI}, 1.050-1.388)$.

Conclusions: This study reveals that thoracic APD reduction and diaphragm elevation are associated with decreased postoperative pulmonary volumes and function in adult PE patients. Nuss procedure for adult patients with PE must be considered cautiously by thoracic surgeons, especially in patients who expect to improve their cardiopulmonary function.

Keywords: Pectus excavatum (PE); Nuss procedure; lung volume measurement; three-dimensional model (3D model)

Submitted Jul 14, 2021. Accepted for publication Aug 06, 2021.

doi: $10.21037 / \mathrm{atm}-21-3822$

View this article at: https://dx.doi.org/10.21037/atm-21-3822 


\section{Introduction}

Pectus excavatum (PE), also known as funnel chest, is a common congenital chest wall deformity occurring between 1 and 8 per 1,000 persons $(1,2)$. Presently, surgical approaches remain the most effective methods for the correction of PE. In 1998, Nuss et al. introduced a minimally invasive procedure that revolutionized the understanding of surgical correction of chest wall deformities (3). Due to its advantages of minimal invasiveness and favorable intraoperative effects (reduced operative time and blood loss) compared to the Ravitch procedure (2), the Nuss procedure has gained increased attention and has been widely adopted.

Although there are many benefits for PE patients that accepted Nuss procedure, some negative surgical outcomes such as recurrence and pleural effusion after Nuss procedure have also been reported in the previous studies $(4,5)$. However, surgeons and researchers paid more attention to the change of cardiopulmonary function after Nuss surgery. Despite the ongoing debate regarding the optimal age for $\mathrm{PE}$ correction, it is widely accepted that $\mathrm{PE}$ correction through the surgical approach should be done before adult age $(6,7)$. Fonkalsrud et al. demonstrated that uncorrected $\mathrm{PE}$ patients presented with worsened symptoms with increasing age and emphasized that both young and adult patients should be surgically treated (8). However, for adult patients with $\mathrm{PE}$ who missed the opportunity to receive surgical treatment during adolescent years, the precise effect of surgical correction on improvement in cardiopulmonary functions remains a much-debated topic. However, some studies have reported that cardiopulmonary function can improve in adult PE patients after Nuss procedure (9-12). Other researchers, however, argued that the primary indication for an adult PE correction is merely cosmetic. Opinions similar to this are supported by various studies reporting little or no improvement in cardiovascular or pulmonary functions following $\mathrm{PE}$ correction, especially for adult patients (13-17).

We here propose a neoteric approach for examining the effects of Nuss procedure on adult PE patients through the application of innovative methods to measure postoperative changes in pulmonary morphology and functions. Therefore, our study aimed at determining the alterations in pulmonary volume and function in adult PE patients and identifying potential predictors associated with the surgical outcomes following PE correction. We present the following article in accordance with the STROBE reporting checklist (available at https://dx.doi.org/10.21037/atm-21-3822).

\section{Methods}

\section{Study population and clinical information}

This was a retrospective cohort study that collected patient data from the Department of Thoracic Surgery, Guangdong Provincial People's Hospital, between January 2017 and December 2019. The PE patients included in the study met the following inclusion criteria: (I) age greater than or equal to 18 years old, (II) having undergone both the first stage Nuss bar placement surgery and the second stage Nuss bar removal surgery in our department, (III) complete preoperative and postoperative computed tomography (CT) data, and (IV) complete follow-up information. Patients with the following characteristics were excluded from the study: (I) aged $<18$ years; (II) no available preoperative or postoperative CT data; and (III) signs of pneumothorax, hemopneumothorax, or inflammation on CT images that could affect the quality of lung reconstruction. Clinical information such as height, weight, body mass index (BMI), age at steel bar insertion, period of Nuss bar placement, and CT measurement value were collected and included in the statistical analysis. A Consolidated Standards of Reporting Trials (CONSORT) diagram was provided to better illustrate the study design (Figure S1).

\section{Three-dimensional (3D) model reconstruction}

CT data in Digital Imaging and Communications in Medicine (DICOM) format was collected using the picture archiving and communication system. Mimics 21.0 (Materialise HQ Technologielaan, Leuven, Belgium) was used to build a 3D model and calculate the lung volume. The chest cages and pulmonary structures were reconstructed at different time points ( $\mathrm{t} 1$ : before bar placement; $\mathrm{t} 2$ : after bar removal), calculated, and compared with the lung volumes (Figure 1). To minimize measurement bias caused by the period between two CT data acquisition points, the manubrium in two copies of CT data sets from each patient was reconstructed and used to confirm that no alterations had occurred in the volume variables (Figure S2).

\section{Acquisition of pulmonary function test (PFT) and ultrasound cardiogram (UCG) results}

The patients' first PFT results during the preoperative evaluation were retrieved. The routine follow-up of PFT took place at the outpatient clinic in the subsequent year. If a patient did not return for follow-up within a year, PFT 

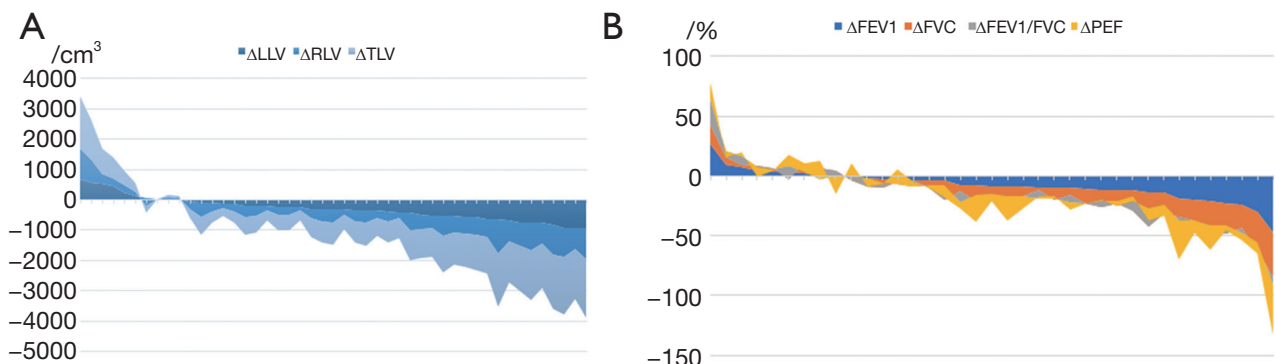

Figure 1 Alterations in adult PE individual pulmonary volumes and functions after Nuss procedure. (A) Alterations in adult PE individual pulmonary volumes. Alterations in individual lung volumes are represented with a stacked area chart. Unilateral lung volume (LLV and RLV) and TLV changes are represented by the different shading of blue area. Decreased lung volumes were observed in the majority of the adult PE patients. (B) Alterations in adult PE individual pulmonary functions. Alterations in individual pulmonary function parameters are represented using a stacked area chart. FEV1 changes are represented by the blue area, FVC changes by the orange area, FEV1/FVC by the gray area, and PEF by the yellow area. Decreased pulmonary functions were observed in majority of the adult patients. PE, pectus excavatum; LLV, left lung volume; RLV, right lung volume; TLV, total lung volume; FEV1, forced expiratory volume in 1 second; FVC, forced vital capacity; PEF, peak expiratory flow.

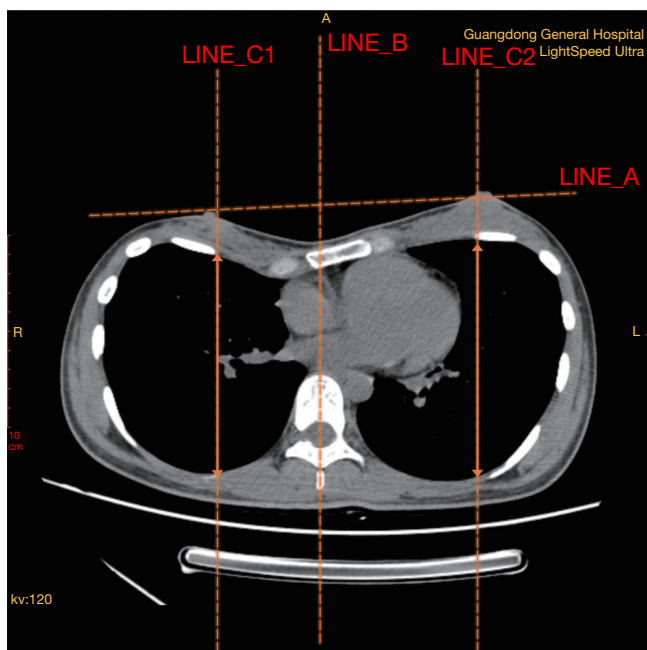

Figure 2 Measurement of the bilateral APD of the thoracic cavity. Line A is formed between two nipples. A fixed transverse plane where Line A is located on the CT images was determined. Line $\mathrm{B}$ was drawn from the midsagittal plane of the thorax which is perpendicular to the anterior median line. Two lines (Line C1, Line C2) parallel to Line B were drawn across the upper border of the fourth ribs. The APD of the thorax was calculated as the length of the intersection between Line $\mathrm{C} 1 / \mathrm{C} 2$ and the thoracic cavity. APD, anteroposterior diameter.

was acquired before their Nuss bar-removal surgery 3 or 5 years later. A standard PFT includes assessing multiple variables and different aspects of pulmonary function.
Forced expiratory volume in 1 second (FEV1), forced vital capacity (FVC), and peak expiratory flow (PEF) were selected for final analyses due to their significant clinical relevance to pulmonary function (18). Left ventricular ejection fraction (LVEF) were measured by UCG.

\section{Measurement of anteroposterior diameter (APD) of the thoracic cavity}

To assess the APD of both the left and right side of the thoracic cavity, we defined the measurement criteria to ensure consistency when obtaining data from different patient files. First, we determined the transverse plane to be where the line formed by bilateral nipples (Line A) was located on CT images. Another line from the midsagittal plane of the thorax that was perpendicular to the anterior median line was drawn as a reference line (Line B). An intersection point of the transverse plane and superior margin of the fourth rib was then identified, and a line was drawn (Line C) parallel to Line B. Finally, the APD of the thoracic cavity was determined as the length of Line $C$ drawn across the thorax. A sketch map (Figure 2) was used to better illustrate the measurement process.

\section{Development of a scoring system for diaphragm position markings}

To evaluate the remodeling in the superoinferior dimension, we reconstructed the diaphragm and the thoracic cage, 

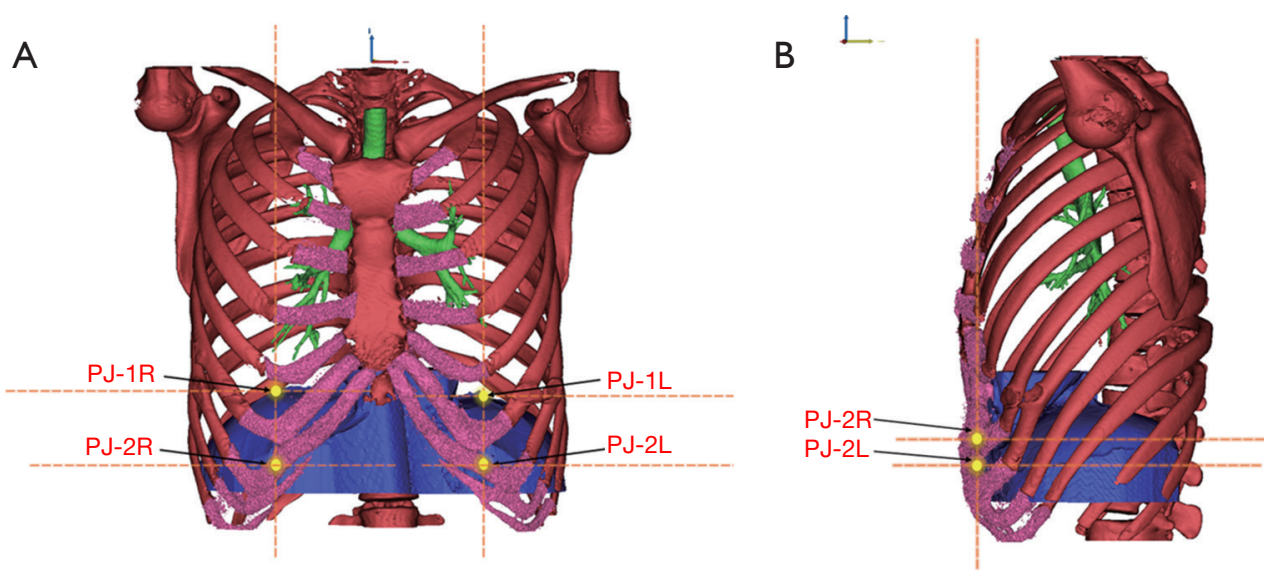

Figure 3 PJs of diaphragm position markings. (A) Localization of bilateral PJ1 scores. The first PJ location is the intersection point between the upper arc of the diaphragm and the ipsilateral midclavicular line in the coronal plane, denoted as "PJ1 score". (B) Localization of the PJ2 scores. The second PJ location is the intersection point between the anterior arc of the diaphragm and the ipsilateral midclavicular line in the sagittal plane, denoted as "PJ2 score". PJ, projection.

which included 12 pairs of ribs, the sternum, xiphoid process, costal cartilages, and 12 thoracic vertebrae. A scoring system was developed to evaluate the relative position between the diaphragm and chest wall (Figure 3). The area of the anterior chest wall was divided into 11 parts. Each part was composed of the area between the upper edge of each rib and that of the next rib. The score of each area was equal to the ordinal number of the rib bone included in that specific area. For example, an area consisting of the first rib and the first intercostal space was denoted as "score 1". After this, we selected two projection (PJ) points from each side of the diaphragm on the respective midclavicular line to describe the relative position of the diaphragm. The first PJ location was the intersection point between the upper arc of the diaphragm and the ipsilateral midclavicular line in the coronal plane and was denoted as "PJ1 score" (Figure 3A). The second PJ location was the intersection point between the anterior arc of the diaphragm and the ipsilateral midclavicular line in the sagittal plane and was denoted as "PJ2 score" (Figure 3B). The average values of the leftand the right-side scores were obtained to represent the final assessment score of the relative diaphragm position. In order to simplify the results regarding the PJ scoring, we averaged the PJ1 and PJ2 scores into a single PJ score. Alteration in $\mathrm{PJ}$ is denoted as $\triangle \mathrm{PJ}$. In on our scoring system, a lower score indicates a higher position of the diaphragm.

\section{Statistical analysis}

Continuous variables of demographic information, such as age, height, and weight, are present as mean \pm standard deviation (SD) or median [interquartile range (IQR)]. Continuous data, such as lung volumes, PJ score, FEV1, FVC, FEV1/FVC and LVEF, were collected before and after Nuss bar placement. Difference between data collected before bar placement and after bar removal were tested using Student's paired $t$-test provided that the data fit a normal distribution. Otherwise, Wilcoxon rank-sum test was performed. Categorical variables were compared by using the chi-square test or Fisher's exact test. The widely accepted Haller index (HI) range for surgical indication of the Nuss procedure is $\mathrm{HI}>3.0(19,20)$ or $\mathrm{HI}>3.25(21-23)$. Subgroup analysis based on the $\mathrm{HI}(\mathrm{HI}<3.0 v s$. $\mathrm{HI} \geq 3.0)$ was performed. Multivariate binary logistic regression models were explored to identify independent predictor variables of altered lung volumes and pulmonary function. The logistic regression models were built through a threestep approach. First, univariate binary logistic regression analyses were performed. Independent variables that were significant $(\mathrm{P}<0.1)$ were included in the subsequent step. Second, collinearity diagnostics for the selected variables were tested. No multicollinearity was identified. Third, multivariate logistic regression models were constructed 
using the selected variables assessed for multicollinearity. A forward stepwise approach was adopted. Potential cofounders, such as sex, BMI, length of bar placement, and preoperative sternal depression depth were included to test their influences on both the independent and dependent variables in the models.

All tests were two-tailed, and the significance level was set at a $\mathrm{P}<0.05$. All statistical analyses were performed using SPSS v.26 software (IBM Corporation, Armonk, NY, USA).

\section{Ethical statement}

This retrospective study was approved by the ethics committee of Guangdong Provincial People's Hospital (No. GDREC2020120H). An electronic written informed consent was obtained from patients who was involved in the research. All procedures performed in this study involving human participants were in accordance with the Declaration of Helsinki (as revised in 2013).

\section{Results}

\section{Demographic information of PE patients}

Out of the 47 included patients, the male to female ratio was approximately 8:1 (42 to 5). The median age was 21 years (IQR: 19-24), with a mean BMI of $18.38 \pm 1.68$. The median HI before the Nuss procedure was 3.70 (IQR: 3.22-4.09). Detailed preoperative descriptive information of the patient population and a summary of the statistical results are presented in Table 1.

\section{Comparison of CT images in 3 years}

In this study, sternal components were reconstructed from two sequential CT data sets of each of the 47 patients, and the manubrium volumes of the built model were compared (Figure S2). Due to the extensive period between placement and removal of the Nuss bar, there was uncertainty on whether the replacement of imaging equipment and technology updates during this period might have affected the measurement accuracy of imaging data. The mean manubrium volume was $23.64 \pm 4.73 \mathrm{~cm}^{3}$ before surgery and $23.70 \pm 4.71 \mathrm{~cm}^{3}$ after surgery. There was no significant difference between the two measured values $(\mathrm{P}=0.117$; Figure $4 A$ ). Therefore, we confirmed that there was no significant volume deviation between the two CT scans.

\section{Preoperative versus postoperative alterations in lung volumes, function, and LVEF}

The lung module of the Mimics 21.0 software was used for lung reconstruction. The left and right lungs were reconstructed (Figure S3), and lung volumes were calculated and compared. Alterations in individual lung volumes and pulmonary functions are presented in the area charts (Figure $1 A, 1 B$ ) Student's paired $t$-test results showed that there was a significant difference between preoperative and postoperative lung volumes in both the left lung ( $L L V, P<0.001)$ and right lung $(\mathrm{RLV}, \mathrm{P}<0.001)$ (Figure $4 B, 4 C$ ). The rate of change in the LLV and RLV were approximately $13.0 \%$. The mean preoperative total lung volume (TLV) of the patients was $4,592.82 \pm 946.54 \mathrm{~cm}^{3}$. After the Nuss procedure, the mean TLV decreased to $3,976.26 \pm 867.35 \mathrm{~cm}^{3}$. The average lung volume decreased by $616.56 \mathrm{~cm}^{3}(\mathrm{P}<0.001)$, which was approximately $13.4 \%$ of the preoperative lung volume (Figure $4 D$ ). No significant difference was observed between the rate of change in the LLV and RLV. A paired sample $t$-test revealed a significant reduction in FEV1 (\%), FVC (\%), and PEF (\%) (Figure $4 E-4 G$ ). Compared with preoperative data, the mean value decreased by $7.8 \%$ in postoperative FEV1 (\%) ( $\mathrm{P}=0.001), 9.0 \%$ in FVC $(\%)(\mathrm{P}=0.001)$, and $4.7 \%$ in $\mathrm{PEF}(\%)(\mathrm{P}=0.023)$. No significant difference was observed between preoperative LVEF $(65.83 \% \pm 3.92 \%)$ and postoperative LVEF $(65.81 \% \pm 4.51 \%)$.

\section{Alterations in postoperative diaphragm location, APD, and anatomical and physiological correlations}

A comparative analysis between the preoperative and postoperative diaphragm position was conducted. The significant upward shift of the diaphragm in both the anterior and top edge of the diaphragm after the operation was observed in the reconstructed model. The preoperative PJ1 score was $5.62 \pm 0.58$, and the score decreased to $5.18 \pm 0.62$ after the operation (Figure $4 H$ ). The PJ2 scores were $6.64 \pm 0.63$ and $6.07 \pm 0.64$ for preoperative and postoperative evaluation, respectively (Figure 4I). The lower the PJ2 score was, the higher the diaphragm elevation observed. Furthermore, the Wilcoxon ranksum test revealed a significant difference in both PJ1 score and PJ2 score before and after the Nuss procedure $(\mathrm{P}<0.001)$, which suggested that significant elevation of the diaphragm occurred after the Nuss procedure. Both the 
Table 1 Pre- and postoperative variables and comparison of results of the studied population

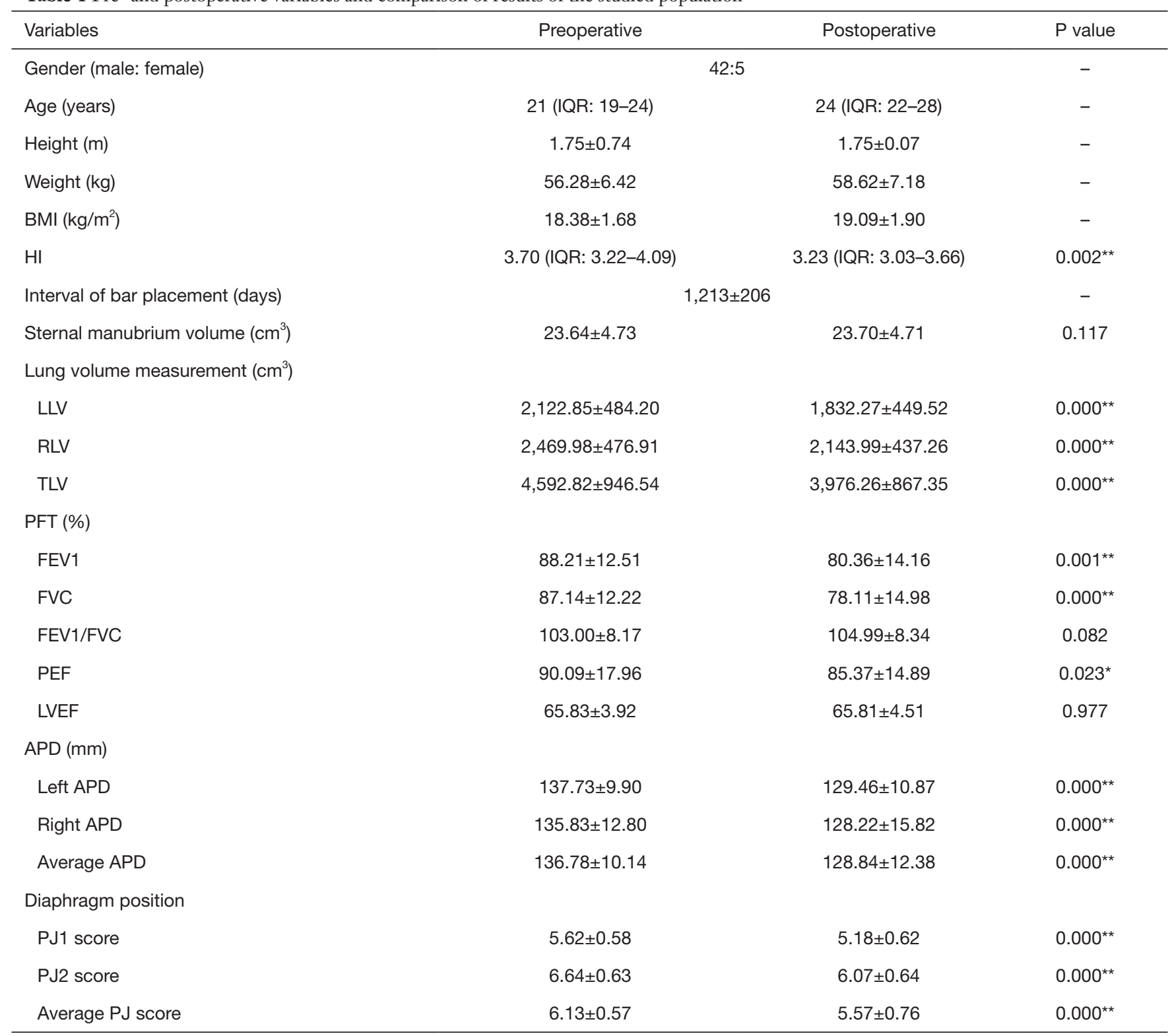

*, $\mathrm{P}<0.05$; ${ }^{* \star}, \mathrm{P}<0.001$. IQR, interquartile range; BMI, body mass index; HI, Haller index; LLV, left lung volume; RLV, right lung volume; TLV, total lung volume; PFT, pulmonary function test; FEV1, forced expiratory volume in 1 second; FVC, forced vital capacity; PEF, peak expiratory flow; LVEF, left ventricular ejection fraction; APD, anteroposterior diameter; PJ, projection.

left and right APD were found to be significantly decreased postoperatively. Paired sample $t$-test showed alterations of $-8.27 \pm 11.01 \mathrm{~mm}(\mathrm{P}<0.001)$ on the left side and $-7.61 \pm$ $11.07 \mathrm{~mm}(\mathrm{P}<0.001)$ on the right side (Figure $47,4 K)$. As a result, the total APD showed a significant decrease after operation (Figure 4L).

To further explore predictors of pulmonary volumes and function changes, binary logistic regression analysis was performed. Of the investigated independent variables, diaphragm elevation was positively associated with decrease in lung volumes [odds ratio $(\mathrm{OR})=40.51 ; \mathrm{P}=0.011 ; 95 \%$ confidence interval (CI), 2.37-692.59]. Patients with a reduction in average APD of the thorax were more likely to obtain a negative pulmonary function result $(\mathrm{OR}=1.21$; 
A

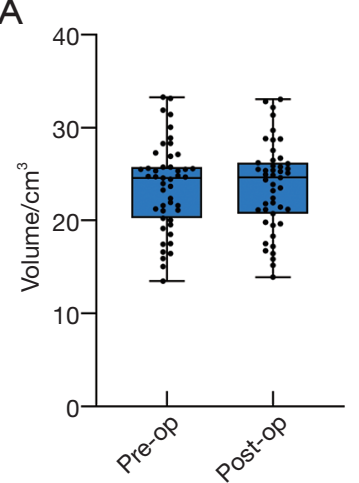

$E$
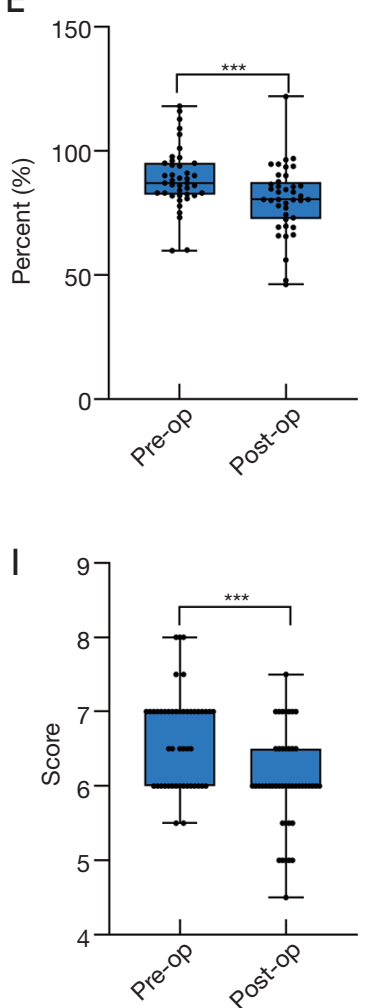

B

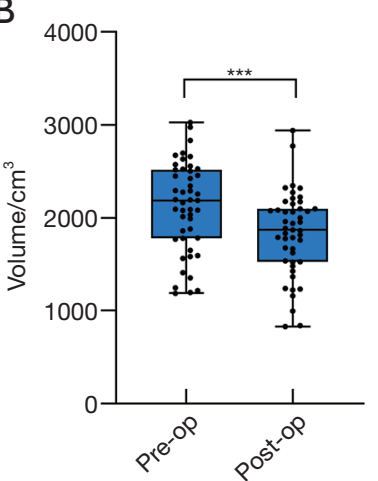

$\mathrm{F}$
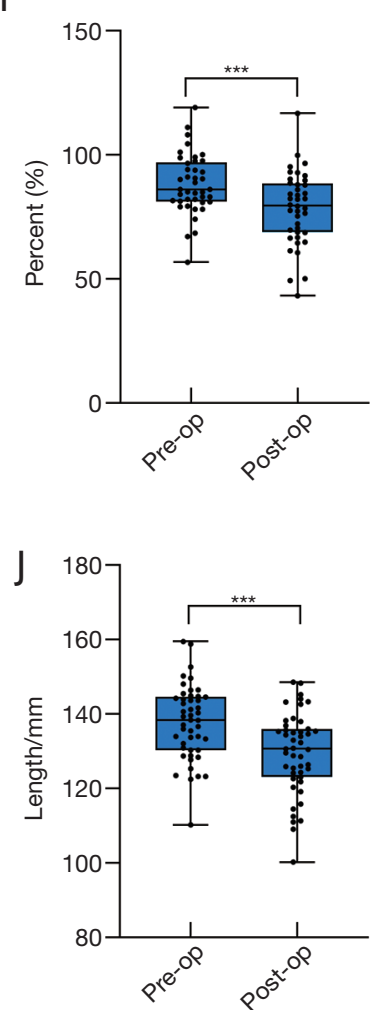

C

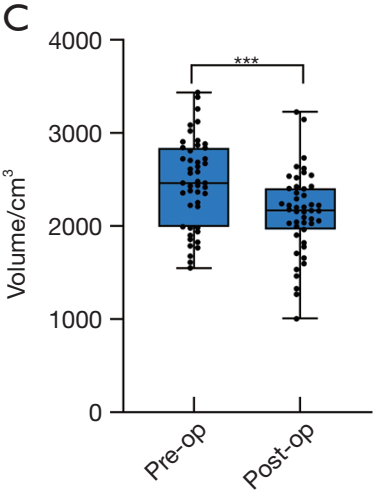

G
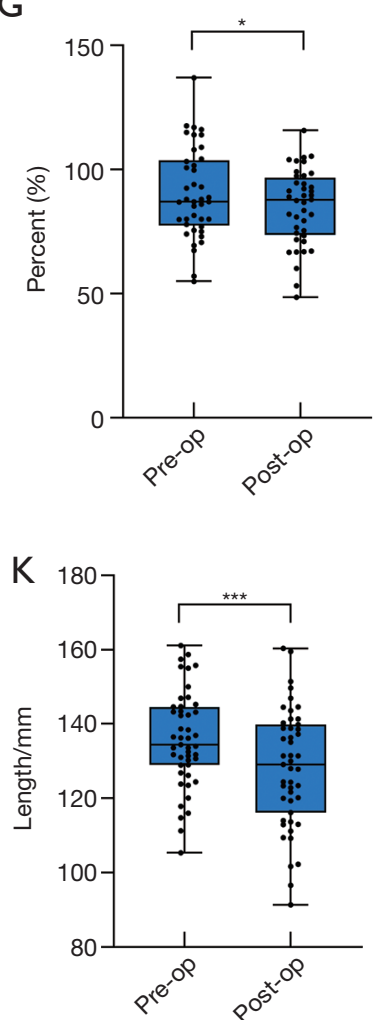

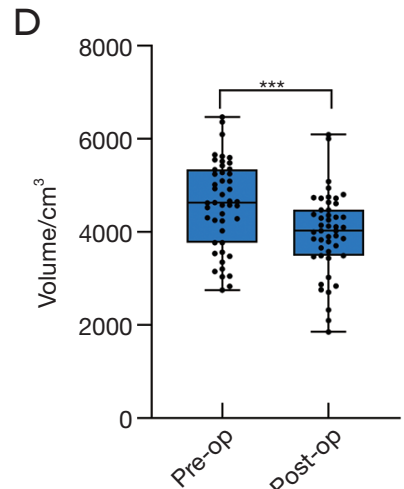

$\mathrm{H}$
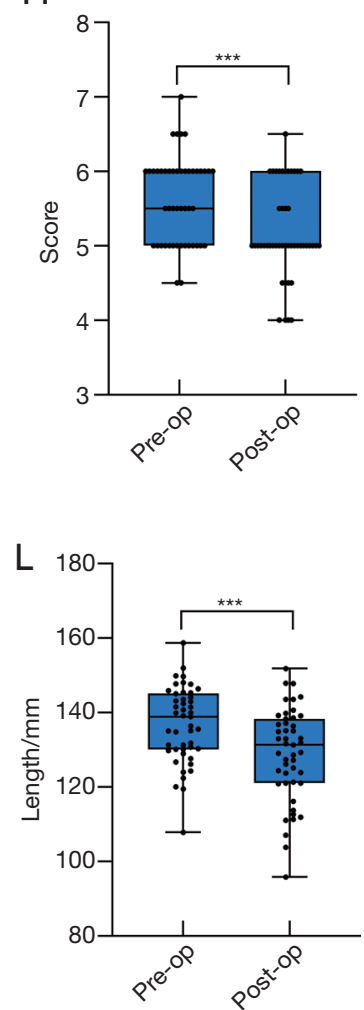

Figure 4 Paired sample test of various variables. (A) Manubrium volume. (B) LLV. (C) RLV. (D) TLV. (E) FEV1. (F) FVC. (G) PEF. (H) PJ1 score. (I) PJ2 score. (J) Left APD. (K) Right APD. (L) Total APD. The upper and lower borders of each box represent the upper and lower quartile, respectively. The middle horizontal line represents the median. The upper and lower whisker represent the largest and smallest values of each measured parameters, respectively. The black dots represent each investigated individual. ${ }^{*}, \mathrm{P}<0.05$; ${ }^{* *}, \mathrm{P}<0.001$. LLV, left lung volume; RLV, right lung volume; TLV, total lung volume; FEV1, forced expiratory volume in 1 second; FVC, forced vital capacity; PEF, peak expiratory flow; APD, anteroposterior diameter. 
$\mathrm{P}=0.008 ; 95 \%$ CI, $1.050-1.388)$.

\section{Subgroup analysis}

Based on the preoperative HI, the included PE patients were divided into two groups ( $\mathrm{HI}<3.0 v s$. $\mathrm{HI} \geq 3.0$ ). Significant differences in alterations were observed between the two HI groups in the LLV $(\mathrm{P}=0.022)$ and in TLV $(\mathrm{P}=0.008)$ (Table $\mathrm{S} 1)$. To further reveal the association between HI and lung volume alterations, continuous variables, including lung volume alterations, were converted into categorical data based on the direction of volume changes (increased or decreased). The chi-squared analysis revealed significant differences in lung volume alterations between the $\mathrm{HI}<3.0$ and $\mathrm{HI} \geq 3.0$ subgroups. The TLV of patients in the higher HI group significantly decreased in size compared to those patients with an HI smaller than 3.0 (94.7\% in $\mathrm{HI} \geq 3.0$ vs. $5.3 \%$ in $\mathrm{HI}<3.0 ; \chi^{2}=6.82$; $\mathrm{P}=0.009<0.05)$. Significant differences were also observed in changes in the $\operatorname{LLV}\left(\chi^{2}=5.64 ; \mathrm{P}=0.018\right)$ and $\operatorname{RLV}\left(\chi^{2}=8.31\right.$; $\mathrm{P}=0.004)$. In terms of PFT, patients with severe chest deformity ( $\mathrm{HI} \geq 3.0$ ) accounted for $89.3 \%$ of those with impaired postoperative FEV1. Similar trends were observed during the analysis of FVC $(89.3 \%$ in $\mathrm{HI} \geq 3.0 \mathrm{vs} .10 .7 \%$ in $\mathrm{HI}<3.0)$ and $\mathrm{PEF}(88.0 \%$ in $\mathrm{HI} \geq 3.0$ vs. $12.0 \%$ in $\mathrm{HI}<3.0)$. Detailed results are presented in Table S2.

\section{Discussion}

Several studies have indicated that the Nuss procedure provides good aesthetic outcomes in adult PE patients and can improve both the physical and psychological healthrelated quality of life over time (24-27). The long-term surgical results also show a high successful orthopaedic effect with low recurrence rate. Fewer than one-tenth of the severe PE patients relapsed after Nuss procedure in our department, and the figure is much lower in patients with mild disease. Especially, all the 47 adult patients in our study obtained favorable operation results without any recurrence. According to our experiences, detailed preoperative assessment leads to the satisfying surgical effects, and implanting multiple Nuss bars or performing osteotomy plus Nuss surgery may also result in better therapeutic effects for severe PE patients. However, these experiences remain to be further verified.

A comparison of pre- and post-operative CT images and $3 \mathrm{D}$ models shows that the sunken sternum was corrected perfectly (Figure 5). However, as earlier indicated, previously published data concerning the effects of $\mathrm{PE}$ correction on cardiopulmonary functions are not consistent. Moreover, little attention has been paid to the changes in pulmonary volumes and pulmonary function.

In this study, the 3D lung model was reconstructed, and the lung volumes of the included 47 adult patients were calculated. The most important finding emerging from the analysis was the alterations in postoperative lung volumes of the adult patients. This study also found that there was a significant reduction in both lung volumes following the Nuss procedure, and the reduction rate was approximately $12 \%$. Similar results were reported in a pediatric study (28). However, this outcome was contradictory to previously published findings, in which the postoperative lung volumes were increased in patients with $\mathrm{PE}$ after the Nuss procedure $(29,30)$. However, studies by Sengul et al. and Redlinger et al. included both children and adults and thus were likely to introduce confounding factors, such as the natural growth and expansion of the children's lungs. Similar to the findings by Malek et al. (14) and Kuru et al. (17), our study demonstrated significant reduction in several PFT variables: $-7.8 \%$ in postoperative FEV1 $(\mathrm{P}=0.001),-9.0 \%$ in $\mathrm{FVC}$ $(\mathrm{P}=0.001)$, and $-4.7 \%$ in $\mathrm{PEF}(\mathrm{P}=0.023)$.

Interestingly, there was no significant difference observed in the FEV1/FVC ratio before and after the Nuss procedure. We assumed that a synchronous rate of change in FEV1 and FVC yielded no significant difference in FEV1/FVC. Furthermore, Lawson et al. reported the main respiratory dysfunction in patients with $\mathrm{PE}$ to be the restrictive pattern in which the FEV1/FVC ratio increased or remained in the normal range (31).

There are several possible explanations for the results presented in the current study. First, compared to children, adult $\mathrm{PE}$ patients have relatively poor chest wall compliance, which poses a challenge for maximal lung expansion during inspiration as well as the contraction during the expiration process. Values of the two pulmonary function representatives, FEV1 and FVC, are closely associated with pulmonary volume (32); hence, reduced lung volumes would further lower FEV1 and FVC. Besides adult PE patients' inherent disadvantages in chest wall compliance, chest wall mobility also affects lung volume and function through a dynamic measurement process.

Second, long-time displacement of the sternocostal articulation is affected by bar placement, and fixation of two lateral stabilizers may gradually force the complete rib cage to change shape. The long-term malalignment of these articulations can cause difficulties in full chest wall mobility, 

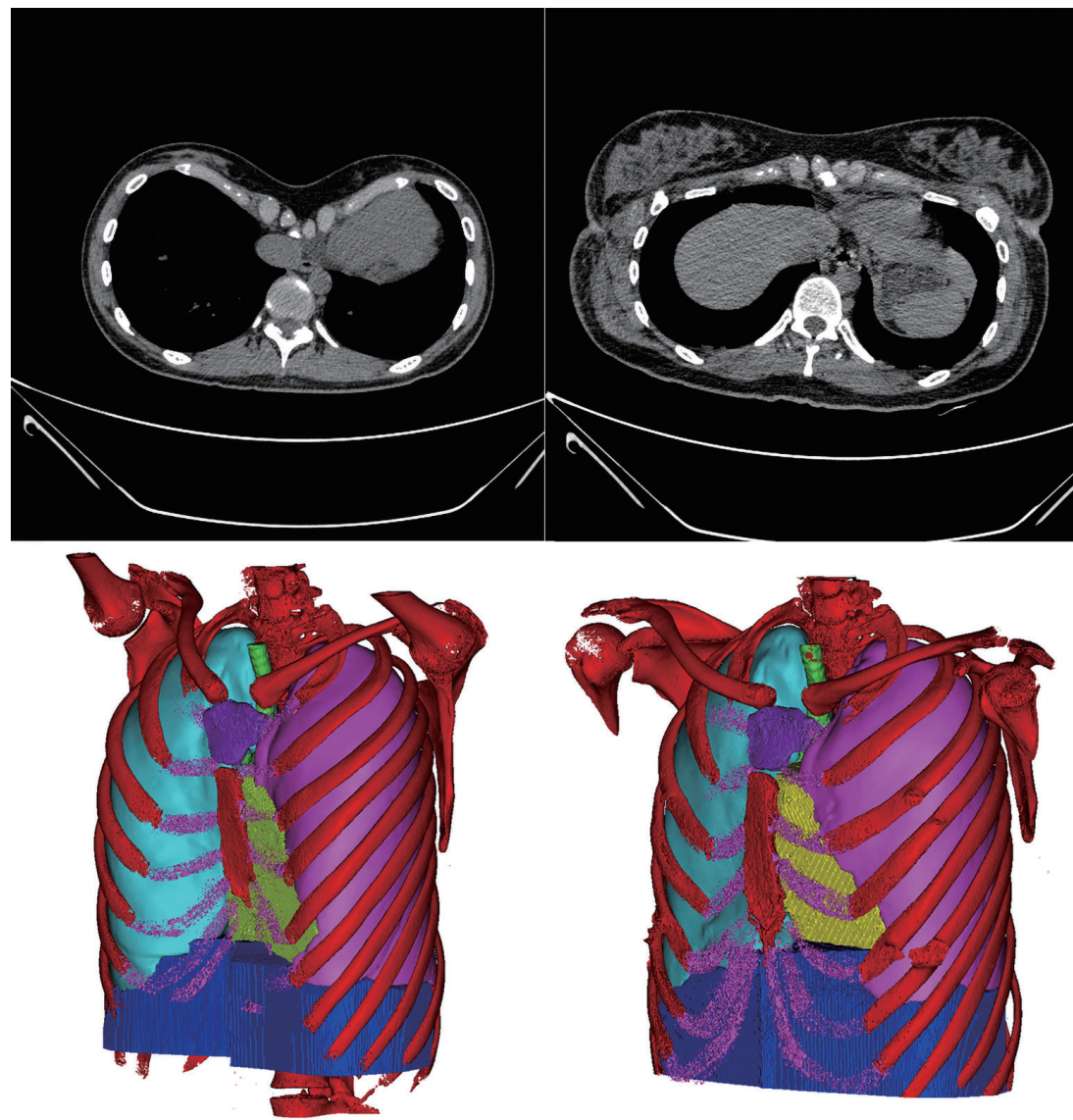

Figure 5 Comparison of CT images and 3D models of a severe PE patient whose pre-operative HI came to 9.10 before and after Nuss procedure. The decrease in APD and the elevation of the diaphragm are quite obvious. (A) Pre-operative CT image. (B) Post-operative CT image. (C) Pre-operative thoracic 3D model. (D) Post-operative thoracic 3D model. CT, computed tomography; 3D, three-dimensional; PE, pectus excavatum; HI, Haller index; APD, anteroposterior diameter.

thus restricting chest wall motion and adversely affecting pulmonary function. For patients with a higher HI, the malalignment of the sternocostal joints appeared to be more evident. This study's hypothesis was supported by Acosta et al., who reported rib cage mobility to be significantly reduced after Nuss procedure for PE (33). A different cohort study reported there to be no change in exercise capacity in patients after Nuss bar removal compared with those with a stiff chest wall embedded with steel (34). These findings demonstrate that a possible decrease in chest wall motion postoperatively could also hinder patients' efforts to expand the lungs to their maximal range. Therefore, poor thoracic compliance and weakened chest wall function after Nuss procedure may contribute to a reduction in lung volume and a deterioration in pulmonary function.

Third, the shortening of both the anteroposterior and the superoinferior axis of the thorax is considered to be another factor. A significant decrease in APD on both sides of the thorax and a positive correlation between the reduction in PFT parameters and reduction in APD were observed. As mentioned earlier, FEV1 and FVC changed with pulmonary volume. The anteroposterior alterations in the thorax, together with other factors, could impede the maximal expansion of the lungs, thus affecting pulmonary function. Moreover, a significant elevation of the diaphragm after the Nuss procedure was observed. Further analysis revealed a positive correlation between the reduction in lung volume and diaphragm elevation. Indeed, the reduced space in the thoracic cavity due to diaphragm elevation could further decrease the pulmonary volume, and the restricted space in the altered lung volumes could significantly affect pulmonary function value. 
Future prospective, multicenter studies should evaluate the advantages and drawbacks of the Nuss procedure on adult $\mathrm{PE}$ patients to assist physicians in making critical decisions while performing minimally invasive surgery on certain adult groups. Although this study successfully demonstrated alterations in pulmonary volumes and functions in adult $\mathrm{PE}$ patients after Nuss procedure, further research is needed to provide a more comprehensive analysis. Future work should determine the reliability of the current study results in different age groups, especially among children and adolescents. Finally, the relatively small sample size of the current study might have introduced a risk of type I error. A study with a larger number of samples from adult PE patients is required to verify this finding in the future.

In conclusion, this study demonstrated a significant decrease in pulmonary volume and function in adult $\mathrm{PE}$ patients after the Nuss procedure was performed. The salient and novel finding of our study was that the decrease in APD and the elevation of the diaphragm can explain the poor surgical outcomes among adult PE patients. Subgroup analysis shows that the negative results were more likely to appear to patients with high HI, which may prompt that severe $\mathrm{PE}$ is one of the predictors of negative surgical results of adult patients. Nuss procedure for adult patients with PE must be considered cautiously by thoracic surgeons, especially in patients who suffered from severe thoracic deformity and expect to improve their cardiopulmonary function.

\section{Acknowledgments}

Funding: None.

\section{Footnote}

Reporting Checklist: The authors have completed the STROBE reporting checklist. Available at https://dx.doi. org/10.21037/atm-21-3822

Data Sharing Statement: Available at https://dx.doi. org/10.21037/atm-21-3822

Conflicts of Interest: All authors have completed the ICMJE uniform disclosure form (available at https://dx.doi. org/10.21037/atm-21-3822). The authors have no conflicts of interest to declare.
Ethical Statement: The authors are accountable for all aspects of the work in ensuring that questions related to the accuracy or integrity of any part of the work are appropriately investigated and resolved. This retrospective study was approved by the ethics committee of Guangdong Provincial People's Hospital (No. GDREC2020120H). An electronic written informed consent was obtained from patients who was involved in the research. All procedures performed in this study involving human participants were in accordance with the Declaration of Helsinki (as revised in 2013).

Open Access Statement: This is an Open Access article distributed in accordance with the Creative Commons Attribution-NonCommercial-NoDerivs 4.0 International License (CC BY-NC-ND 4.0), which permits the noncommercial replication and distribution of the article with the strict proviso that no changes or edits are made and the original work is properly cited (including links to both the formal publication through the relevant DOI and the license). See: https://creativecommons.org/licenses/by-nc-nd/4.0/.

\section{References}

1. Obermeyer RJ, Goretsky MJ. Chest wall deformities in pediatric surgery. Surg Clin North Am 2012;92:669-84, ix.

2. Toci GR, Davis TA, Bigelow BF, et al. Analyzing outcomes of nuss and ravitch repair for primary and recurrent pectus excavatum in adults. Ann Thorac Surg 2020;110:272-5.

3. Nuss D, Kelly RE Jr, Croitoru DP, et al. A 10-year review of a minimally invasive technique for the correction of pectus excavatum. J Pediatr Surg 1998;33:545-52.

4. Cho DG, Kim JJ, Park JK, et al. Recurrence of pectus excavatum following the Nuss procedure. J Thorac Dis 2018;10:6201-10.

5. Cheng YL, Lin CT, Wang HB, et al. Pleural effusion complicating after Nuss procedure for pectus excavatum. Ann Thorac Cardiovasc Surg 2014;20:6-11.

6. Nuss D, Kelly RE Jr. Indications and technique of Nuss procedure for pectus excavatum. Thorac Surg Clin 2010;20:583-97.

7. Nuss D. Minimally invasive surgical repair of pectus excavatum. Semin Pediatr Surg 2008;17:209-17.

8. Fonkalsrud EW, Dunn JC, Atkinson JB. Repair of pectus excavatum deformities: 30 years of experience with 375 patients. Ann Surg 2000;231:443-8. 
9. Chao CJ, Jaroszewski DE, Kumar PN, et al. Surgical repair of pectus excavatum relieves right heart chamber compression and improves cardiac output in adult patients--an intraoperative transesophageal echocardiographic study. Am J Surg 2015;210:1118-24; discussion 1124-5.

10. Krueger T, Chassot PG, Christodoulou M, et al. Cardiac function assessed by transesophageal echocardiography during pectus excavatum repair. Ann Thorac Surg 2010;89:240-3.

11. Neviere R, Benhamed L, Duva Pentiah A, et al. Pectus excavatum repair improves respiratory pump efficacy and cardiovascular function at exercise. J Thorac Cardiovasc Surg 2013;145:605-6.

12. Töpper A, Polleichtner S, Zagrosek A, et al. Impact of surgical correction of pectus excavatum on cardiac function: insights on the right ventricle. A cardiovascular magnetic resonance study $\dagger$. Interact Cardiovasc Thorac Surg 2016;22:38-46.

13. Malek MH, Berger DE, Housh TJ, et al. Cardiovascular function following surgical repair of pectus excavatum: a metaanalysis. Chest 2006;130:506-16.

14. Malek MH, Berger DE, Marelich WD, et al. Pulmonary function following surgical repair of pectus excavatum: a meta-analysis. Eur J Cardiothorac Surg 2006;30:637-43.

15. Aronson DC, Bosgraaf RP, Merz EM, et al. Lung function after the minimal invasive pectus excavatum repair (Nuss procedure). World J Surg 2007;31:1518-22.

16. Udholm S, Maagaard M, Pilegaard H, et al. Cardiac function in adults following minimally invasive repair of pectus excavatum. Interact Cardiovasc Thorac Surg 2016;22:525-9.

17. Kuru P, Dudakli A, Mursaloglu H, et al. How pulmonary function changes after pectus excavatum correction surgery. Asian Cardiovasc Thorac Ann 2015;23:945-9.

18. van Dalen C, Harding E, Parkin J, et al. Suitability of forced expiratory volume in 1 second/forced vital capacity vs percentage of predicted forced expiratory volume in 1 second for the classification of asthma severity in adolescents. Arch Pediatr Adolesc Med 2008;162:1169-74.

19. Goldstein SD, Colombani PM. Preoperative assessment of chest wall deformities. In: Saxena AK. editor. Chest wall deformities. Heidelberg: Springer Berlin Heidelberg, 2017:191-5.

20. Abu-Tair T, Turial S, Hess M, et al. Impact of pectus excavatum on cardiopulmonary function. Ann Thorac Surg 2018;105:455-60.

21. Kelly RE, Goretsky MJ, Obermeyer R, et al. Twenty- one years of experience with minimally invasive repair of pectus excavatum by the Nuss procedure in 1215 patients. Ann Surg 2010;252:1072-81.

22. Sesia SB, Heitzelmann M, Schaedelin S, et al. Standardized haller and asymmetry index combined for a more accurate assessment of pectus excavatum. Ann Thorac Surg 2019;107:271-6.

23. Poston PM, Patel SS, Rajput M, et al. The correction index: setting the standard for recommending operative repair of pectus excavatum. Ann Thorac Surg 2014;97:1176-9; discussion 1179-80.

24. Hanna WC, Ko MA, Blitz M, et al. Thoracoscopic Nuss procedure for young adults with pectus excavatum: excellent midterm results and patient satisfaction. Ann Thorac Surg 2013;96:1033-6; discussion 1037-8.

25. Krasopoulos G, Dusmet M, Ladas G, et al. Nuss procedure improves the quality of life in young male adults with pectus excavatum deformity. Eur J Cardiothorac Surg 2006;29:1-5.

26. Zuidema WP, Oosterhuis JWA, Zijp GW, et al. Early consequences of pectus excavatum surgery on selfesteem and general quality of life. World J Surg 2018;42:2502-6.

27. Sacco Casamassima MG, Gause C, Goldstein SD, et al. Patient satisfaction after minimally invasive repair of pectus excavatum in adults: long-term results of nuss procedure in adults. Ann Thorac Surg 2016;101:1338-45.

28. Shimoyama S, Kobayashi T, Inoue Y, et al. Left displacement of the mediastinum determines the imbalance in the pulmonary vascular bed and lung volume in children with pectus excavatum. Pediatr Surg Int 2008;24:549-53.

29. Sengul AT, Sahin B, Celenk C, et al. The effect of minimally invasive surgical repair on the lung volumes of patients with pectus excavatum. Thorac Cardiovasc Surg 2014;62:226-30.

30. Redlinger RE Jr, Wootton A, Kelly RE, et al. Optoelectronic plethysmography demonstrates abrogation of regional chest wall motion dysfunction in patients with pectus excavatum after Nuss repair. J Pediatr Surg 2012;47:160-4.

31. Lawson ML, Mellins RB, Paulson JF, et al. Increasing severity of pectus excavatum is associated with reduced pulmonary function. J Pediatr 2011;159:256-61.e2.

32. Qian QZ, Cao XK, Shen FH, et al. Correlations of smoking with cumulative total dust exposure and cumulative abnormal rate of pulmonary function in coalmine workers. Exp Ther Med 2016;12:2942-8.

33. Acosta J, Bradley A, Raja V, et al. Exercise improvement 
Page 12 of 12

after pectus excavatum repair is not related to chest wall function. Eur J Cardiothorac Surg 2014;45:544-8.

34. Oswald N, Jalal Z, Kadiri S, et al. Changes in chest wall motion with removal of Nuss bar in repaired

Cite this article as: Gao Z, Huang S, Tang Y, Wang S, Zhuang W, Ding Y, Wu H, Tang J, Zhang D, Zhou H, Ben X, Xie L, Chen G, Qiao G. Factors influencing negative surgical outcomes in adult pectus excavatum patients undergoing Nuss procedure. Ann Transl Med 2021;9(16):1335. doi: 10.21037/ atm-21-3822

\section{Gao et al. Poor results after Nuss surgery in adult PE}

pectus excavatum - a cohort study. J Cardiothorac Surg 2019;14:4.

(English Language Editor: J. Gray) 


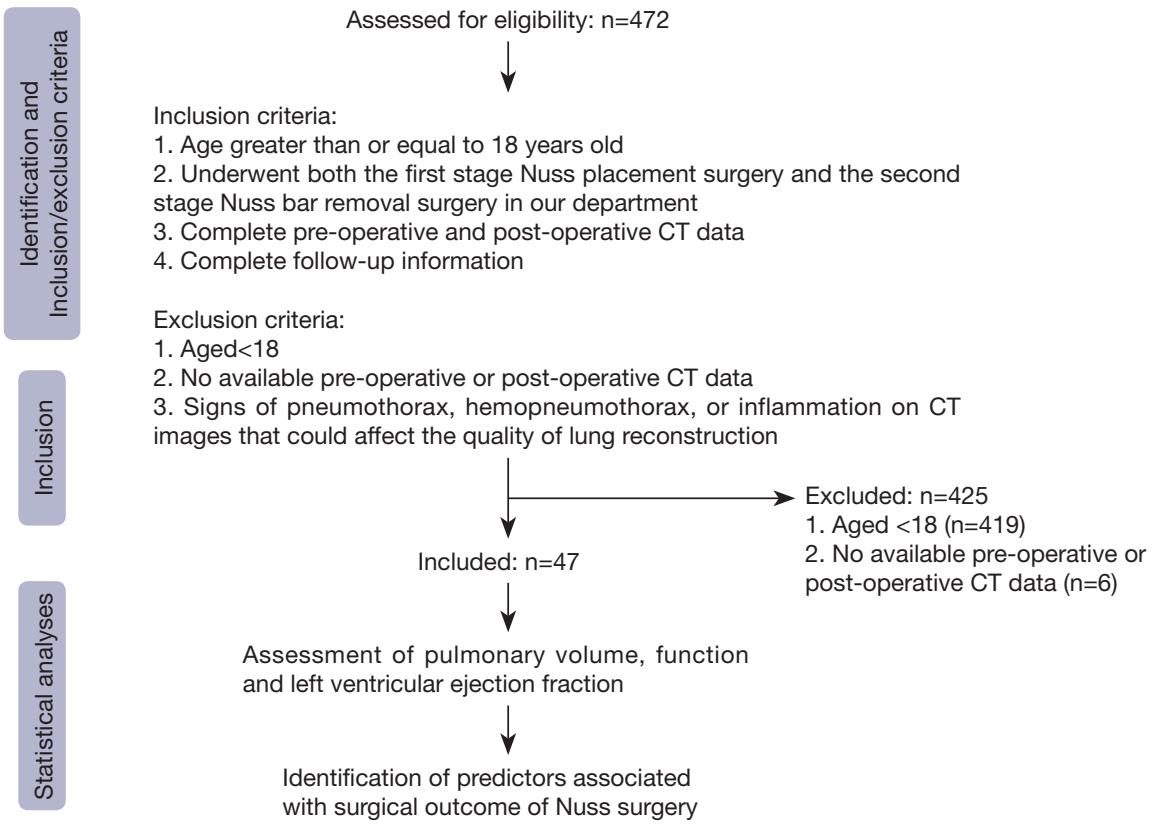

Figure S1 CONSORT diagram of the study design. CONSORT, Consolidated Standards of Reporting Trials.
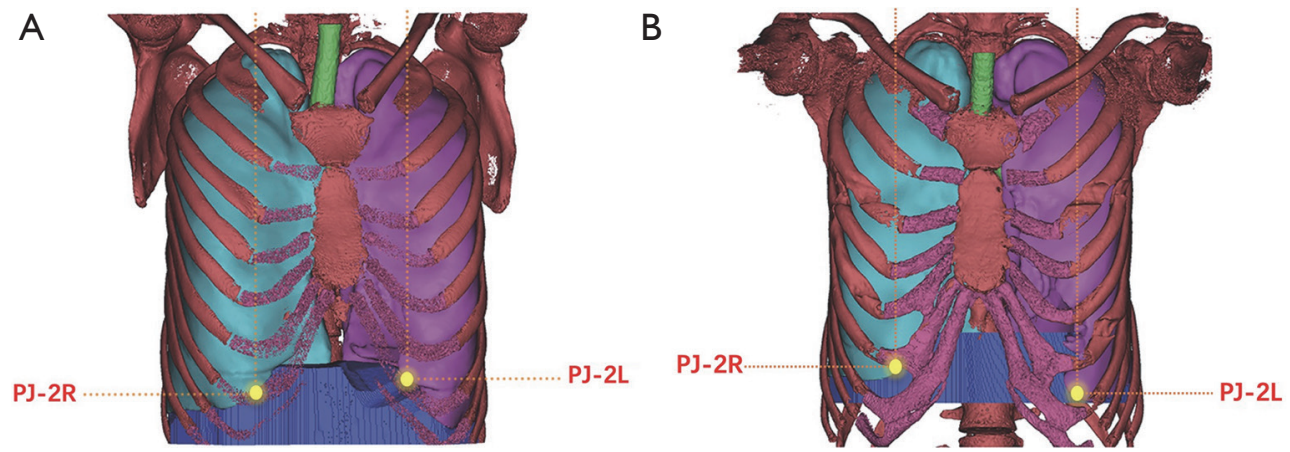

Figure S2 Reconstruction and comparison of the pre- and postoperative sternum. (A) Reconstruction of the preoperative sternum. (B) Reconstruction of the postoperative sternum. PJ, projection.

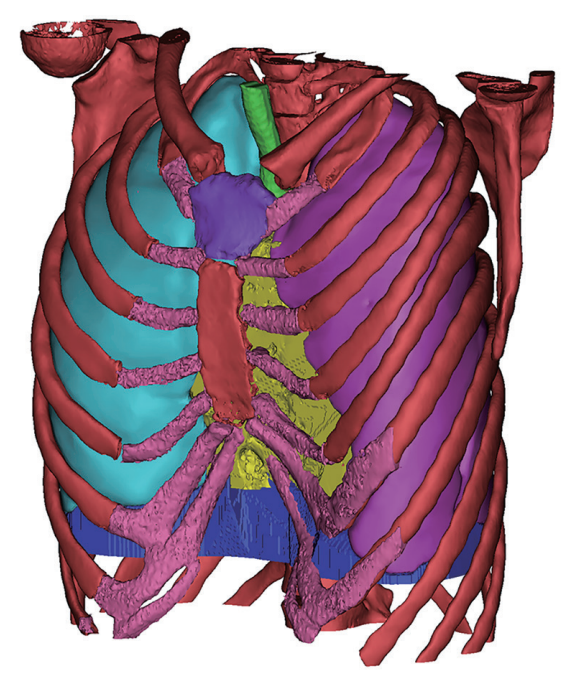

Figure S3 Reconstruction of 3D thorax model. 3D, threedimensional. 
Table S1 Comparison of lung volumes between the two $\mathrm{HI}$ groups $(\mathrm{HI}<3.0$ vs. $\mathrm{HI} \geq 3.0)$

\begin{tabular}{|c|c|c|c|c|}
\hline Variables & $\begin{array}{l}\text { Mean } \pm \text { SD } \\
\mathrm{HI}<3.0(n=6)\end{array}$ & $H I \geq 3.0(n=41)$ & $P$ value & $95 \% \mathrm{Cl}$ \\
\hline$\Delta \mathrm{LLV}$ & $41.59 \pm 551.64$ & $-339.19 \pm 337.92$ & $0.022^{*}$ & 56.94 to 704.62 \\
\hline$\triangle \mathrm{RLV}$ & $130.63 \pm 699.72$ & $-392.81 \pm 333.16$ & 0.128 & -209.01 to $1,255.90$ \\
\hline$\triangle T L V$ & $172.23 \pm 1,250.36$ & $-732.00 \pm 654.10$ & $0.008^{\star \star}$ & 248.95 to 1559.51 \\
\hline
\end{tabular}

* $\mathrm{P}<0.05$; ** $\mathrm{P}<0.001$. HI, Haller index; SD, standard deviation; Cl, confidence interval; LLV, left lung volume; RLV, right lung volume; TLV, total lung volume.

Table S2 Association between $\mathrm{HI}$ and alterations in lung volumes and functions

\begin{tabular}{|c|c|c|c|c|}
\hline \multirow{2}{*}{ Variables } & \multicolumn{2}{|c|}{ HI subgroups } & \multirow{2}{*}{$c^{2}$-statistic [df] } & \multirow{2}{*}{$\mathrm{P}$ value } \\
\hline & $<3.0, \mathrm{n}$ (\% within variable) & $\geq 3.0, \mathrm{n}$ (\% within variable) & & \\
\hline $\operatorname{LLV}\left(\mathrm{cm}^{3}\right)$ & & & $5.64[1]$ & $0.018^{*}$ \\
\hline Increase & $4(40.0)$ & $6(60.0)$ & & \\
\hline Decrease & $2(5.4)$ & $35(94.6)$ & & \\
\hline $\operatorname{RLV}\left(\mathrm{cm}^{3}\right)$ & & & $8.31[1]$ & $0.004^{* *}$ \\
\hline Increase & $4(50.0)$ & $4(50.0)$ & & \\
\hline Decrease & $2(5.1)$ & $37(94.9)$ & & \\
\hline $\operatorname{TLV}\left(\mathrm{cm}^{3}\right)$ & & & $6.82[1]$ & $0.009^{* *}$ \\
\hline Increase & $4(44.4)$ & $5(55.6)$ & & \\
\hline Decrease & $2(5.3)$ & $36(94.7)$ & & \\
\hline FEV1 (\%) & & & $0.101[1]$ & 0.75 \\
\hline Increase & $2(22.2)$ & $7(77.8)$ & & \\
\hline Decrease & $3(10.7)$ & $25(89.3)$ & & \\
\hline FVC (\%) & & & $0.101[1]$ & 0.75 \\
\hline Increase & $2(22.2)$ & $7(77.8)$ & & \\
\hline Decrease & $3(10.7)$ & $25(89.3)$ & & \\
\hline PEF (\%) & & & $0.151[1]$ & 0.698 \\
\hline Increase & $2(12.0)$ & $10(88.0)$ & & \\
\hline Decrease & $3(16.7)$ & $22(83.3)$ & & \\
\hline
\end{tabular}

*, $\mathrm{P}<0.05 ;{ }^{* *}, \mathrm{P}<0.001 . \mathrm{HI}$, Haller index; LLV, left lung volume; RLV, right lung volume; TLV, total lung volume; FEV1, forced expiratory volume in 1 second; FVC, forced vital capacity; PEF, peak expiratory flow. 